\title{
乎
}

Andoni Maiza Larrarte*

Ricardo Bustillo Mesanza**

\section{ANÁLISIS DEL MODELO DE INTERNACIONALIZACIÓN EN CHINA DE LOS FABRICANTES EUROPEOS DE AUTOMÓVILES}

La mayoría de los principales países productores de vehículos de Europa occidental ha padecido un recorte en las cifras de producción y empleo del sector desde el comienzo de la crisis. Sin embargo, la industria germana ha conseguido mantener sus niveles de actividad, en parte, gracias a los positivos resultados alcanzados en el mercado chino. El objetivo del artículo consiste en analizar las razones del éxito de las empresas alemanas a partir del examen del modelo de internacionalización. El estudio de la literatura, de los datos de comercio exterior y de las experiencias empresariales permite concluir que los productores germanos han sabido abordar el mercado chino con una estrategia mejor definida, basada en su avanzada tecnología y sus buenas prácticas de gestión, y con un mayor contenido de producción doméstica.

Palabras clave: fragmentación internacional, comercio exterior, inversión extranjera.

Clasificación JEL: F23, F10, F62.

\section{Introducción: la industria de automoción europea y el «factor chino»}

La consolidación de nuevos mercados de enormes dimensiones y rápido crecimiento representa, junto a la evolución hacia las energías y modos de conducción alternativos, lo que algunos autores han dado en llamar como «la segunda revolución de la automoción» (Freyssenet, 2009). En ese contexto, China es uno de los mercados de mayor tamaño y el que ha registrado mayores tasas de crecimiento durante la última década. En concreto, la matriculación de vehículos ha alcanzado los 22

* Economía Aplicada I. Universidad del País Vasco, UPV/EHU. Escuela de Estudios Empresariales de Vitoria-Gasteiz.

** Economía Aplicada V. Universidad del País Vasco, UPV/EHU. Facultad de Ciencias Económicas y Empresariales.

Versión de mayo de 2015. millones de unidades en 2013, es decir, 16 millones más que en 2005.

La demanda china se ha visto impulsada por un creciente segmento de población de clase media que adquiere su primer vehículo, ya que en su actual fase de desarrollo la sociedad del país se sitúa en un tramo de elevada elasticidad de demanda-renta (Haugh et al., 2010). Lo que es más, probablemente esa evolución se mantendrá a corto y medio plazo, ya que la tasa de motorización es todavía relativamente baja y conserva un amplio margen de recorrido al alza.

Paralelamente, gracias a la estrategia diseñada por las autoridades públicas, la República Popular China (RPC) se ha convertido en el país que produce un número más elevado de vehículos de pasajeros. Ciertamente, China representa también en este apartado el caso más destacado de una tendencia más general, ya que recientemente $\triangleright$ 


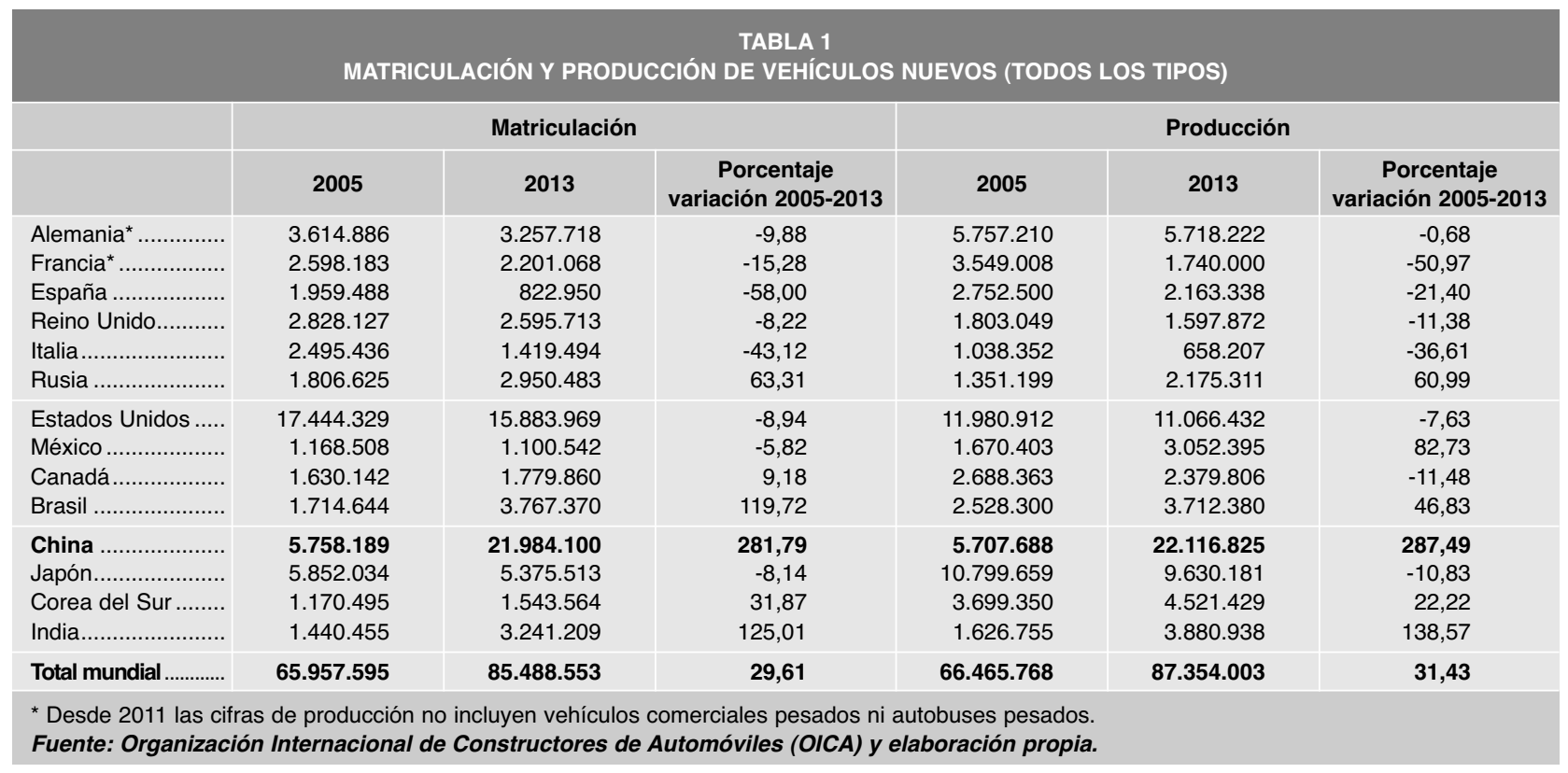

la producción ha registrado considerables incrementos en otras grandes economías como India, Brasil y Rusia.

Por el contrario, la industria del automóvil ha padecido recortes en la mayor parte de las economías desarrolladas y, concretamente, en los países de Europa occidental, los Estados Unidos y Japón, economías todas ellas con niveles de renta notablemente más elevados y con tasas de motorización cercanas a niveles de saturación.

Cabe destacar, no obstante, que en el seno de la Unión se aprecian diferencias reseñables, ya que mientras en Alemania se mantienen cifras de producción similares a las de los ejercicios de bonanza económica, en el resto de los principales países productores, especialmente Francia, Italia y España, tanto la producción como el empleo han sufrido importantes descensos.

En un entorno marcado por un posible problema de sobrecapacidad productiva ${ }^{1}$, la habilidad para penetrar en mercados exteriores representa la principal alternativa a la «solución» de acometer desinversiones. Hay varios ejemplos recientes de cierres de planta en Europa (Opel en

\footnotetext{
1 Algunos estudios, basándose en la tasa de motorización, el PIB per cápita, el crecimiento estimado, etcétera, apuntan la existencia de un problema de «sobrecapacidad» principalmente en Italia, España y Francia (Haugh et al., 2010; OCDE, 2013).
}

Antwerp-Bélgica, Fiat en Termini-Italia, etcétera), o de sustanciales recortes en las plantillas por el traslado de la producción hacia mercados emergentes (Nicholls, 2013), y el nivel de competencia entre centros de producción europeos alcanza niveles muy elevados ${ }^{2}$.

Considerando que el sector de la automoción «conserva una importancia estratégica y representa un pilar de la industria y la economía europea, al proporcionar empleos de calidad a millones de trabajadores de la UE», y con el objetivo de crear «una base industrial sólida para producir vehículos de transporte por carretera y sus componentes» (Comisión Europea, 2012a y 2012b), resulta de sumo interés entender las razones que han permitido a Alemania obtener un comportamiento significativamente más positivo.

Por ello, el principal objetivo de este artículo consiste en analizar la posible incidencia del «factor chino" sobre los productores europeos de vehículos. A tal efecto, en los siguientes apartados se aborda el papel desempeñado por la fragmentación internacional de la producción en la automoción y se reflexiona sobre la estrategia de $D$

\footnotetext{
2 Por ejemplo, en un informe encargado por una asociación de fabricantes y comerciantes de vehículos del Reino Unido, se concluye que «la planta de Nissan en Sunderland tiene que competir con plantas de Renault en Francia y en España, así como con otras plantas de Nissan para garantizar la asignación de futuros modelos» (KPMG, 2014).
} 
ANÁLISIS DEL MODELO DE INTERNACIONALIZACIÓN EN CHINA DE LOS FABRICANTE EUROPEOS...

\begin{tabular}{|c|c|c|c|c|}
\hline \multicolumn{5}{|c|}{$\begin{array}{l}\text { TABLA 2 } \\
\text { EMPLEO EN EL SECTOR DEL AUTOMÓVIL EN EUROPA* (2004-2013) } \\
\text { (Miles de trabajadores) }\end{array}$} \\
\hline & \multicolumn{2}{|c|}{ NACE Rev. 1.1} & \multicolumn{2}{|c|}{ NACE Rev. 2} \\
\hline & 2004 & 2008 & 2008 & 2013 \\
\hline Alemania & $1.032,3$ & $1.135,2$ & $1.119,2$ & $1.097,5$ \\
\hline 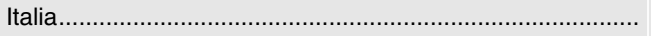 & 231,6 & 223,3 & 234,9 & 210,1 \\
\hline Francia & 323,5 & 359,6 & 345,9 & 223,4 \\
\hline España & 246,7 & 223,8 & 233,0 & 181,5 \\
\hline Reino Unido & 266,6 & 225,7 & 234,2 & 188,7 \\
\hline
\end{tabular}

penetración en China de las principales empresas europeas.

\section{La fragmentación internacional de la producción en el sector del automóvil}

La fragmentación internacional de la producción implica la creación de una cadena de valor añadido que disemina las fases del proceso de producción en regiones distantes del planeta. Este modelo de fabricación se ha extendido debido a múltiples razones, si bien siguiendo a Jones y Kierzkowsky (1990) las multinacionales eligen esta opción cuando se consigue una substancial reducción de los costes de producción sin que por ello se disparen los costes de los servicios que conectan las diferentes fases productivas ${ }^{3}$.

La fragmentación internacional se ha reflejado en un gran incremento del volumen de comercio de bienes intermedios, y numerosos estudios han facilitado estadísticas sobre la importancia de la especialización vertical a través del incremento del comercio intraindustrial vertical (Feenstra y Hanson, 2001; Hummels et al., 2001; Chen et al., 2005; Kimura y Ando, 2005), o mediante datos de comercio de tráfico de perfeccionamiento activo y pasivo ${ }^{4}$ (Görg, 2000; Egger y Egger, 2005; Bustillo y Maiza, 2012).

${ }^{3}$ Estos servicios incluyen tanto factores hardware (aeropuertos, puertos, carreteras, electricidad, telecomunicaciones, etcétera), como software (cargas aduaneras, restricciones a las importaciones, etcétera).

4 Los regímenes de perfeccionamiento activo y pasivo (RPA y RPP) conceden exenciones fiscales consistentes en exenciones/reducciones de cargas tarifarias a los bienes importados/exportados para continuar con su procesamiento industrial y volver a ser exportados/importados.
El sector del automóvil comparte características relevantes con otras industrias: el rápido crecimiento de la inversión extranjera directa y el comercio internacional, la externalización nacional e internacional, el surgimiento de poderosas multinacionales proveedoras ${ }^{5}$, etcétera (Turkcan y Ates, 2010). No obstante, el sector también presenta algunos rasgos distintivos.

En primer lugar, un elevado grado de concentración empresarial6; la industria de la automoción es altamente intensiva en capital y los productores utilizan diversas estrategias para mantener a raya sus costes de producción. Por ejemplo, utilizan una única plataforma para producir varios modelos, colaboran con otras empresas para adquirir tecnología e invertir en I+D, etcétera (Coffin et al., 2013).

En segundo lugar, el ensamblado último de los vehículos se produce habitualmente cerca del mercado final, en parte, debido a las presiones ejercidas sobre los fabricantes para «producir allí donde se vende" (Sturgeon et al., 2008). La sensibilidad ante un elevado volumen de importaciones, especialmente en regiones en las que existe un productor doméstico relevante, frecuentemente conduce a los responsables políticos y a las propias empresas a tener en cuenta el factor de «contenido mínimo local» en su estrategia ${ }^{7}$.

\footnotetext{
${ }^{5}$ Los fabricantes de primer nivel (TIER-1) elaboran sistemas, subsistemas y componentes que, por lo general, están completamente terminados, disponen de alta tecnología y suministran directamente al fabricante de vehículos.

6 Aunque la irrupción de las grandes empresas chinas e indias ha reducido el poder de las compañías hegemónicas; de los tres grandes productores de vehículos (GM, Toyota y Volkswagen), únicamente el fabricante alemán incrementó su producción entre 2007 y 2011.

7 El éxito de los fabricantes japoneses en el mercado estadounidense les llevó a establecer límites de exportación "voluntarios» y, posteriormente, a establecer sus empresas de producción en EEUU (Sturgeon y Florida, 2004).
} 
En tercer lugar, estrechamente unido al factor anterior, las estructuras productivas regionales y nacionales conservan una fortaleza sorprendente cuando se compara con otras industrias de bienes intensivas en volumen (Carrillo et al., 2004; Dicken, 2007), de forma que habitualmente la producción de piezas suele destinarse a plantas de ensamblaje de la región ${ }^{8}$. Durante los últimos años los proveedores han asumido un rol más relevante en el diseño, y una ubicación cercana a los clientes se presenta como un factor crítico para lograr una colaboración más estrecha.

No obstante, la capacidad para producir en todas las regiones del mundo se ha convertido en una especie de «precondición» para poder optar a algunos pedidos (Sturgeon et al., 2008; Sturgeon et al., 2009), y en la actualidad la mayoría de los principales proveedores suministran a los mismos clientes por todo el mundo y se ocupan de realizar los ajustes necesarios en sus componentes para cumplir los requisitos específicos de cada uno de los mercados.

A este respecto, el cuarto y último factor característico es un grado de estandarización de piezas y componentes relativamente bajo, ya que las piezas y subsistemas tienden a ser específicos para modelos concretos. Este hecho se encuentra ligado a la existencia de factores locales notablemente dispares (condiciones de las carreteras, gustos específicos de los consumidores, tamaño familiar, etcétera), pero también a la escasa predisposición que muestran los fabricantes para colaborar y crear unos estándares de producción más extendidos (Sturgeon et al., 2009).

\section{Internacionalización de las empresas europeas del automóvil en China}

En 2013 la UE obtuvo un superávit de 27.000 millones en su balanza comercial con China en el

\footnotetext{
8 En 2006 la producción y ventas de los principales fabricantes europeos y estadounidenses estaban fuertemente concentradas en su región (55 por 100 como media), y sólo en el caso de los fabricantes japoneses se apreciaba un mayor grado de diversificación (Sturgeon et al., 2009).
}

sector de la automoción ${ }^{9}$, resultado alcanzado en gran medida gracias al intenso crecimiento de las exportaciones. Ese dato es extraordinario y contrasta con el déficit global de 131.000 millones de euros que la UE soporta con aquel país, así como con el déficit de 5.300 millones de dólares de los Estados Unidos en el comercio de automoción con la RPC ${ }^{10}$.

En todo caso, hay que reseñar que las cifras de comercio de los diferentes países de la UE-27 arrojan un balance muy dispar. Destaca, en positivo, el crecimiento de las exportaciones de Alemania, magnitud que en 2013 asciende a 18.400 millones de euros, es decir, un 60 por 100 de las ventas totales europeas (el superávit alemán asciende a 17.775 millones de euros, 62 por 100 del europeo).

Del total de exportaciones germanas aproximadamente un 60 por 100 (11.000 millones de euros) corresponde a vehículos ya terminados ${ }^{11}$ (CUCl781, 782 y 783) y el restante 40 por 100 corresponde a piezas y componentes fabricados por la industria auxiliar (CUCI 784, 7132 y 7783), producción en gran medida asociada con el posterior ensamblaje de vehículos en joint-ventures con participación germana.

En esta línea, cabe reseñar que Volkswagen es el fabricante europeo que ha conseguido un mayor éxito en China. Tras la regulación adoptada en $1985^{12}$ Volkswagen puso en marcha una joint venture con Shanghai Automotive Corporation (SAIC) que con el tiempo se ha convertido, junto a la iniciativa conjunta entre General Motors y SAIC, en líder de un $D$

\footnotetext{
9 A los efectos del análisis de comercio exterior, el sector del automóvil se ha definido según la clasificación utilizada por la OMC (OMC, 2014) y la Comisión Europea (Comisión Europea, 2014c), y comprende las siguientes categorías $\mathrm{CUCl}$ : 7132, 7783, 781, 782, 783 y 784 (véase Tabla A.1 del Anexo). Según la clasificación CNAE habitualmente se utiliza el código CNAE 34 para el sector del automóvil (Comisión Europea, 2009).

10 El déficit de 2011 es resultado de un superávit de 5.300 millones de dólares en vehículos y un déficit de 10.500 millones de dólares en partes y componentes (Departamento de Comercio de los Estados Unidos, 2012).

11 Cerca del 100 por 100 de las exportaciones alemanas de bienes finales corresponden al código CUCI 78120 «Vehículos automotores para el transporte de personas», es decir, vehículos de pasajeros.

12 Los productores de automóviles extranjeros pueden fabricar vehículos en China únicamente si crean una joint venture y solo algunas de las joint ventures reciben la aprobación del Gobierno. En ese proceso las autoridades chinas fomentan la transferencia de tecnología y el establecimiento de centros de I+D en China (Nam, 2011).
} 
ANÁLISIS DEL MODELO DE INTERNACIONALIZACIÓN EN CHINA DE LOS FABRICANTE EUROPEOS...
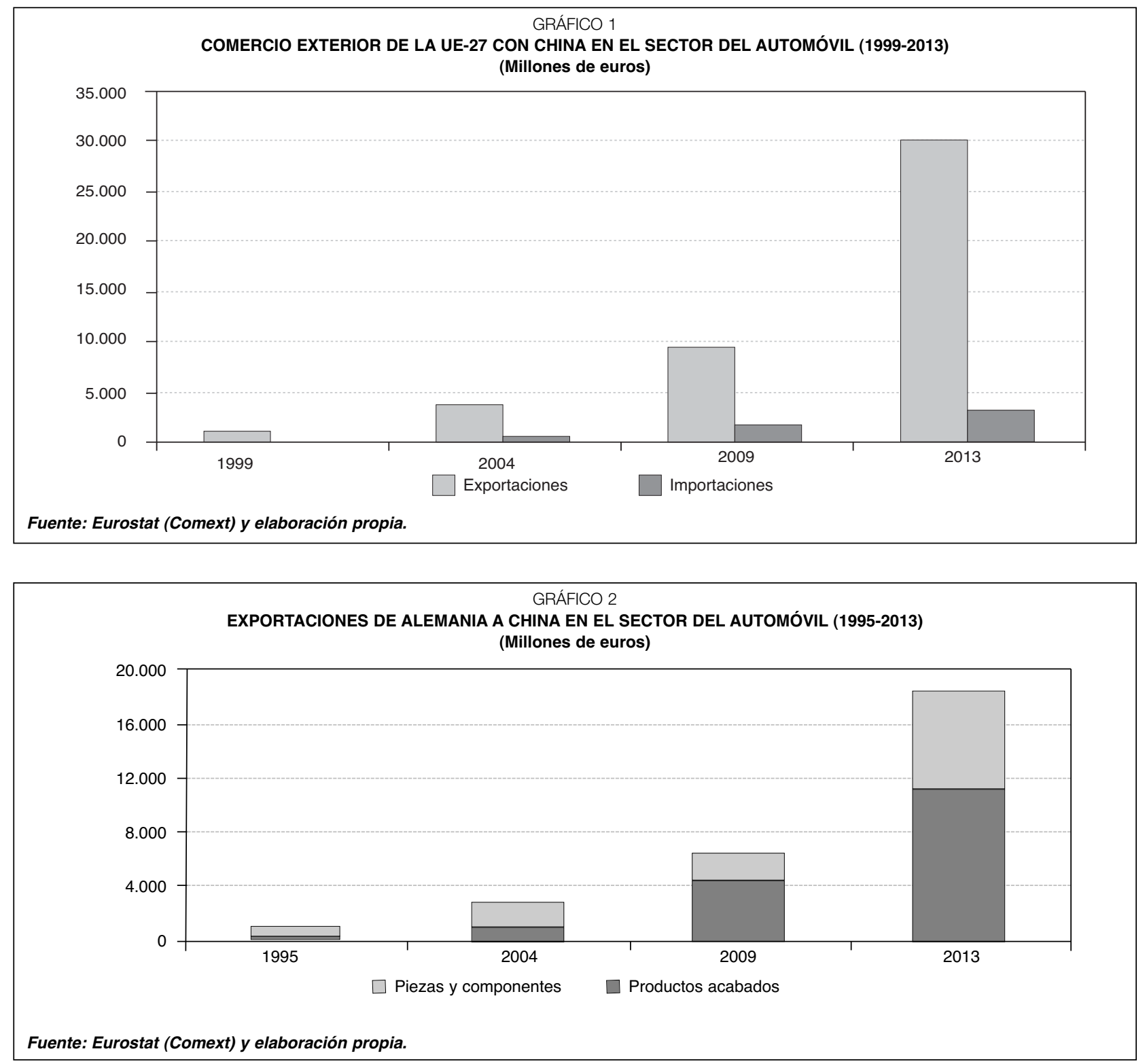

mercado en el que compiten más de 100 fabricantes chinos, muchos de ellos participados por el Gobierno central o los Gobiernos locales (Tabla A.2 del Anexo).

Aún más importante, Volkswagen ha mantenido un volumen relativamente elevado de producción doméstica que posteriormente es transportada al mercado chino ${ }^{13}$. En 2013 Volkswagen vendió 3

13 La reducida proporción de ventas en el país de origen se debe al relativamente pequeño tamaño del mercado alemán. La proximidad con el resto de países europeos, donde Volkswagen vendió 2,96 millones de las unidades producidas en 2011 (un 24,5 por 100 del total), es probablemente la razón por la que esta empresa continúa produciendo una proporción tan grande de sus vehículos en Alemania (Coffin et al., 2013). millones de vehículos producidos en China ${ }^{14}$, y las entregas a clientes en aquel mercado, incluyendo importaciones, alcanzaron un total de 3,3 millones de unidades (Volkswagen, 2014).

La ventaja de Alemania respecto al resto de países europeos es muy notable. Sólo el Reino Unido alcanza un volumen de exportaciones reseñable con 5.500 millones de euros en $2013(18,2$ por $100 \triangleright$

14 La presencia del Grupo Volkswagen comprende más de 60 modelos. Además de los modelos tradicionales, el grupo produce otros que han sido específicamente adaptados al mercado chino (por ejemplo, con una distancia entre ejes mayor), y vehículos específicamente diseñados para clientes chinos como el Volkswagen Lavida, el New Bora y los modelos Santana (Volskwagen, 2014). 


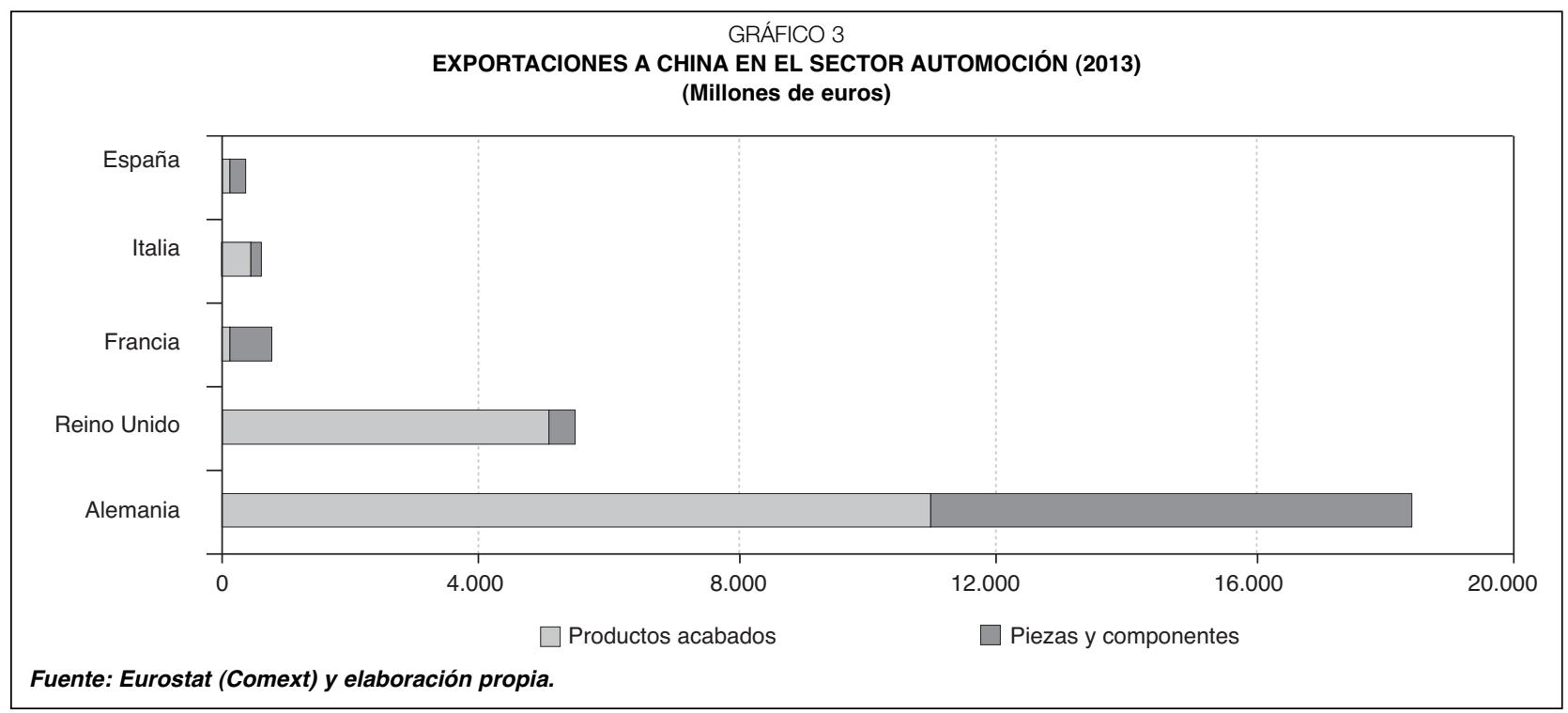

\section{TRAYECTORIA DEL GRUPO VOLKSWAGEN EN CHINA}

- Shanghai-Volkswagen (SVW) fue fundada en 1985 y comenzó sus operaciones con el ensamblaje de kits para el modelo Santana, un sedán de segmento medio basado en tecnología de un VW de 1982.

- Durante los 5 primeros años SVW utilizó las instalaciones remodeladas de la antigua Shanghai Tractor and Automobile Corporation (STAC), que representaron la contribución "en especie" de SAIC a la joint venture. La fábrica STAC inicial era muy intensiva en mano de obra y tenía una producción anual muy limitada (5.000 vehículos).

- En noviembre de 2013 salió de la línea de producción de la fábrica el vehículo 10 millones y en la actualidad China representa un pilar del grupo.

- En 2013 se pusieron en marcha 5 nuevas instalaciones en el país: una nueva planta de vehículos Shanghai-Volkswagen en Urumqui (pionera en el oeste de China), una planta para la producción del nuevo Skoda Superb in Ningbo, la joint venture FAW-Volkswagen lanzó la producción del nuevo Golf en Foshan, una planta de motores fue inaugurada en Changchun, y una nueva planta de componentes en Foshan.

- Al concluir 2013 el Grupo Volkswagen reunía un total de 17 fábricas para la producción de vehículos y componentes.

Fuente: Volkswagen (2014) y Posth (2006) y elaboración propia.

del total europeo), si bien estas ventas corresponden en gran medida a empresas de capital extranjero implantadas en el Reino Unido ${ }^{15}$. En 2013, más de tres cuartas partes de los vehículos fabricados en las islas británicas fueron exportados, ocupando

\footnotetext{
15 La japonesa Nissan es, con diferencia, el principal fabricante de vehículos del Reino Unido con 502.000 coches en 2013. Las marcas británicas Jaguar Land Rover (340.000 coches) y MINI (175.000) ocupan el segundo y cuarto puesto, respectivamente, si bien Jaguar es propiedad del grupo indio Tata Motors y MINI lo es del grupo germano BMW. Toyota y Honda completan el cuadro de principales productores presentes en el Reino Unido.
}

China, con un 10 por 100 , el primer lugar entre los destinos fuera de la UE (SSMT, 2014).

Por otra parte, y a diferencia de lo que sucede con Volkswagen, los fabricantes de Italia, Francia y España apenas utilizan su capacidad doméstica para atender la demanda de la RPC. Tanto las empresas francesas como las italianas han creado joint ventures en China (PSA y Renault-Nissan con Dongfeng Motor Corporation y Fiat con GAC), y las exportaciones se mantienen en niveles muy bajos: 800 millones de euros de Francia, 650 millones de Italia, y 375 millones de España en 2013.

Según Chiappini (2011) los fabricantes alemanes han ganado cuota de mercado en el exterior porque todavía producen más en su país de origen que en el extranjero, mientras que los pobres resultados de las empresas francesas e italianas se deben, principalmente, a la relocalización de la producción en los países emergentes. De tal modo, la relocalización habría causado una reducción de las exportaciones francesas, mientras que la estrategia de fragmentar las fases productivas habría permitido a las empresas alemanas mantener cifras de exportación más elevadas.

En la actualidad China es percibida por los fabricantes europeos principalmente como un enorme mercado que se atiende a través de exportaciones y producción local (joint ventures), y los datos de comercio de bienes intermedios y piezas y componentes reflejan que la creación de una cadena $\triangleright$ 


\section{TRAYECTORIA DE PEUGEOT-CITROËN EN CHINA}

- Peugeot Citroën llegó a China en 1985. Guangzhou Peugeot Automobile Company (GPA) fue creada como una joint-venture con el Gobierno de Guangzhou, pero los resultados fueron decepcionantes.

- Al principio Peugeot introdujo su sistema de gestión, controlaba las decisiones clave, promovió la cultura de empresa e incluso enseñaba francés a los empleados chinos.

- Sin embargo, cuando la competencia se intensificó, surgieron diversos problemas y Peugeot fue acusada de producir modelos caros y antiguos. Además, graves conflictos culturales y luchas internas agravaron la situación.

- En el periodo 1985-1996 se produjeron solamente 100.000 coches, y las pérdidas alcanzaron 2,9 billones de yuanes. En 1996 GPA prácticamente había paralizado su producción.

- Sin embargo, en la actualidad China es el segundo mayor mercado del grupo PSA (sólo por detrás de Francia). En 2013 las ventas del grupo se incrementaron un 26,1 por 100 hasta 557.000 unidades (442.000 en 2012), y la cuota de mercado era del 3,64 por 100.

- En julio de 2013 PSA puso en marcha la tercera planta de Dongfeng Peugeot Citroën Automobiles (DPCA), la joint venture del grupo francés con el fabricante Dongfeng

Fuente: PSA (2014) y Fernández y Shengjun (2007) y elaboración propia.

de valor sino-europea en el sector del automóvil dista de ser una realidad.

En Estados Unidos, por el contrario, ante el incremento de competencia en el mercado doméstico de empresas extranjeras, los principales productores locales (General Motors, Ford y Daimler-Chrysler) han adquirido un elevado grado de dependencia de proveedores de la RPC con el fin de reducir sus costes de producción ${ }^{16}$ (Klier y Rubenstein, 2006).

En este sentido, se ha de reseñar que el escenario estadounidense no parece tan improbable para los países europeos, ya que los flujos de comercio del sector se encuentran muy condicionados por las decisiones de unos pocos grandes fabricantes. Así, unas pocas operaciones de inversión podrían fortalecer la presencia europea en China, la presencia china en Europa o ambas al mismo tiempo, lo que probablemente conduciría a un modelo más dependiente de la fragmentación internacional de la producción.

\footnotetext{
16 Se habría producido un esquema de integración vertical hacia atrás, distinto al europeo o, en concreto, al alemán, donde predomina un grado de integración débil pero hacia adelante, dada la elevada exportación de componentes a China.
}

\section{VOLVO CARS - GEELY SWEDEN AB}

- En 1999 la sueca Volvo Group decidió separarse en dos empresas: Volvo Group, fabricante de vehículos comerciales y, Volvo Cars, fabricante de automóviles.

- Posteriormente Volvo Cars fue adquirida por Ford, pero los resultados no fueron positivos, y se produjeron varios años de pérdidas y despidos masivos reiterados.

- Con la llegada de la crisis Ford decidió vender Volvo Cars, y fue la empresa china Geely Holding Group la que absorbió al fabricante sueco en octubre de 2009.

- La nueva empresa, Geely Sweden AB, ha obtenido resultados financieros positivos durante los últimos ejercicios (EBITDA de 9.826 millones de euros en 2013), con un volumen creciente de ventas en el mercado chino (61.146 unidades en 2013)

- La expansión en China ha sido promovida mediante la creación de dos joint ventures en Daqing y Zhangjiakou (planta de motores), y el comienzo de la producción en la filial de Chengdu.

Fuente: Volvo Cars (2014) y elaboración propia.

\section{Razones del éxito de las empresas alemanas fabricantes de automóviles en China}

Tal vez cabría esperar que los turismos de bajo precio parcialmente producidos en China representaran la base de la estrategia de negocio de la industria europea, porque ello sería coherente con un mercado integrado principalmente por consumidores de baja renta per capita. No obstante, el éxito de los fabricantes germanos parece indicar que han sido modelos más lujosos y caros los que han encontrado su nicho de mercado ${ }^{17}$.

Hay varias razones que explican el éxito germano. En primer lugar, las empresas alemanas, no sólo las del sector del automóvil, tienen una larga trayectoria en la RPC. De hecho, muchas de ellas han padecido severos problemas debido a la complejidad de las relaciones con aquel país (Teijelo, 2009), pero han seguido insistiendo $\triangle$

\footnotetext{
17 Los vehículos exportados desde Alemania y el Reino Unido, al igual que los de Estados Unidos y Japón, incluyen más modelos de lujo destinados a segmentos de población con ingresos altos, mientras que los vehículos fabricados por las firmas locales suelen ser más baratos y están destinados a habitantes con un menor poder adquisitivo (Coffin et al., 2013). El valor de los turismos exportados por Alemania ronda los 23 euros $/ \mathrm{kg}$. (alrededor de 32.000 euros un turismo de $1.400 \mathrm{~kg}$.), mientras que la ratio de los vehículos importados apenas alcanza los 6-7 euros/kg.
} 


\begin{tabular}{|c|c|c|c|}
\hline RELEVANCIA DEL MERC & $\begin{array}{r}\text { TABLA } \\
\text { NO PARA LAS E }\end{array}$ & EAS DEL AU & \\
\hline & \multirow{2}{*}{$\begin{array}{l}\text { Ventas totales } \\
\text { (unidades) }\end{array}$} & \multicolumn{2}{|c|}{ Ventas en China } \\
\hline & & Unidades & Porcentaje total \\
\hline 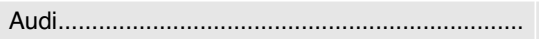 & 1.751 .007 & 491.989 & 28,1 \\
\hline BMW & 1.969 .380 & 391.700 & 19,9 \\
\hline 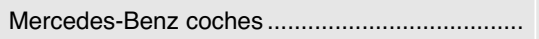 & 1.565 .600 & 239.000 & 15,3 \\
\hline 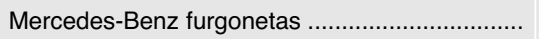 & 270.144 & 12.705 & 4,7 \\
\hline Volkswagen - turismos ${ }^{\star}$ & 8.957 .975 & 3.266 .235 & 36,5 \\
\hline Volkswagen - vehículos comerciales ${ }^{\star} \ldots \ldots \ldots \ldots \ldots . . . .$. & 772.705 & 4.868 & 0,6 \\
\hline Grupo PSA & 2.818 .000 & 557.000 & 19,8 \\
\hline 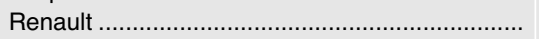 & 2.628 .208 & 34.000 & 1,3 \\
\hline FIAT Spa ${ }^{\star \star}$ & 4.409 .500 & 90.000 & 2,0 \\
\hline
\end{tabular}

después de cada revés, han aprendido valiosas lecciones de gestión intercultural y hoy en día cuentan con joint ventures exitosas en la automoción, en la industria química y en la máquina herramienta (Sohm et al., 2009).

En segundo lugar, algunas de las grandes compañías alemanas tuvieron una mejor intuición sobre la relevancia de la RPC y una «estrategia para China» más clara. Además, las empresas del automóvil tuvieron el apoyo y la compañía de otros pioneros alemanes en su aventura ${ }^{18}$ y las economías del conocimiento o de gestión asociadas al tamaño de la economía alemana (redes formales e informales, recursos, etcétera) han resultado sin duda muy relevantes en este éxito.

Tercero, muchas de las joint ventures creadas en China pretenden que los fabricantes locales aprendan de sus socios extranjeros (o que incluso adquieran su tecnología), y a este fin las iniciativas con los socios alemanes se han priorizado frente a otras opciones europeas dada la imagen de «tecnología avanzada» de los productos germanos en la RPC ${ }^{19}$. Inicialmente sólo unas pocas empresas multinacionales recibieron permiso para invertir en casos especiales (Chen, 2003) y, además, en determinados momentos críticos hubo

\footnotetext{
18 Siemens fue la primera empresa extranjera en invertir en la RPC y ya en 1995 las exportaciones de Alemania superaban la suma de las de Italia y Francia.

19 En 2012 los tres fabricantes europeos con mayor volumen de inversión en I+D eran alemanes: Volkswagen con 11.700 millones de dólares, Daimler con 7.400 millones y BMW con 5.200 millones. A continuación en el ranking se situaban PSA con 2.700 millones, Renault con 2.500 millones y Fiat con 2.400 millones (Coffin et al., 2013).
}

fabricantes estadounidenses y japoneses que no estuvieron dispuestos a arriesgar su tecnología, lo que abrió las puertas a las empresas alemanas $^{20}$ (Leng, 2004).

Y, por último, la relevancia estratégica que Alemania otorga a los mercados internacionales queda evidenciada por el carácter exportador de su economía, y el sector del automóvil representa un claro ejemplo de este hecho. Tal y como se afirma en el Informe Anual de 2013 de la Asociación Alemana de la Industria de Automoción (VDA, 2014):

«La industria de automoción alemana está donde quiera que están los mercados. Esta estrategia ha resultado exitosa nuevamente en el ejercicio pasado. Nuestros fabricantes y proveedores están ampliando su campo de acción global y fueron capaces de beneficiarse de la creciente economía que gira en torno a la automoción fuera de la Europa occidental. El mercado global de vehículos creció un 4 por 100 en 2012, hasta 68 millones de unidades. El mercado chino fue el principal responsable de ese crecimiento, junto a la recuperación del mercado estadounidense. Los fabricantes alemanes se beneficiaron de esta evolución e incrementaron su cuota de mercado en China al 22 por 100 y también ganaron cuota de mercado en los Estados Unidos, por séptimo año consecutivo».

\footnotetext{
20 En el caso concreto del automóvil los otros dos grandes fabricantes con los que los chinos tuvieron negociaciones en los setenta, General Motors y Toyota respondieron «gracias, pero no», y así fue como Volkswagen llegó a China (Posth, 2006).
} 


\section{ANÁLISIS DEL MODELO DE INTERNACIONALIZACIÓN EN CHINA DE LOS FABRICANTE EUROPEOS...}

\section{Conclusiones}

Entre los grandes cambios que vive la industria del automóvil algunos son comunes a los de la economía global y se encuentran relacionados con la irrupción de las grandes economías emergentes. Otros rasgos, por el contrario, son característicos de este sector: una gran concentración de poder en unos pocos fabricantes, un proceso de regionalización que acompaña al de globalización y un débil grado de estandarización de los productos fabricados.

La tendencia a la regionalización implica que los coches son ensamblados allí donde son producidos. Este factor condiciona el futuro de las fábricas situadas en los países de la Europa occidental dada la madurez de estos mercados (elevados niveles de motorización y crisis económica), y el contraste con el rápido crecimiento de enormes mercados como el de China e India.

Italia, Francia y España han sufrido una reducción en los niveles de producción y empleo durante la última década. Por el contrario, los resultados obtenidos en Alemania y, en menor medida, en Reino Unido, han sido significativamente más positivos. Se ha demostrado que el mayor volumen de exportaciones a los países emergentes $y$, en particular, al mercado chino, es uno de los motivos de la fortaleza de la industria germana.

Por el momento las firmas alemanas han descartado el modelo de regionalización imperante y no han trasladado su producción a la RPC; al contrario, han optado por exportar un número relativamente elevado de vehículos terminados ( $\mathrm{y}$ también de piezas y componentes), lo que parece haber sido clave para salvar el descenso registrado en las ventas de mercados más maduros. Así, en 2013 las exportaciones de Alemania representaron algo más del 60 por 100 del total de las ventas de la UE-27 en China.

La penetración en un mercado como el chino constituye un reto y una potencial solución para mitigar el impacto de los posibles problemas de sobrecapacidad existentes en Europa, pero podría no ser suficiente. Todas las empresas del sector, también las alemanas, están ampliando su capacidad de producción en la RPC, y no parece claro que un modelo de producción en el que los vehículos y/o sus componentes primero son fabricados en Alemania y posteriormente son exportados continúe siendo válido en el futuro.

Las empresas italianas y francesas han optado por satisfacer la demanda china principalmente a través de joint-ventures con socios locales, y el contenido europeo en la fabricación de esos modelos es bastante reducido. Más aún, tal y como apunta la experiencia de los Estados Unidos, conviene vigilar el potencial impacto de un creciente volumen de importaciones de bajo coste procedente de China.

\section{Bibliografía}

[1] AUDI (2014). Annual Report, 2013.

[2] BMW Group (2014). Annual Report, 2013.

[3] BUSTILLO, R. y MAIZA, A. (2012). «An analysis of the economic integration of China and the European Union: the role of European trade policy», Asia Pacific Business Review, vol. 18, Issue 3, pp. 355-372.

[4] CARRILLO, J.; LUNG, Y. y VAN TULDER, R., (eds.) (2004). Cars Carriers of Regionalism? New York: Palgrave Mac Millan.

[5] CHEN, X. (2003). From Political Alliance in China's Conception to Comprehensive Partnership in Building: the relations between China and the European Community/European Union. Dissertation.

[6] CHEN, H.; KONDRATOWICZ, M. y YI K.M. (2005). «Vertical specialization and three facts about U.S. international trade». The North American Journal of Economics and Finance, vol.16, pp. 35-59.

[7] CHIAPPINI, R. (2011). "Offshoring and export performance in the European automotive industry». Competition and change, vol. 16, Issue 4 (October 2012), pp. 323-342.

[8] COFFIN, D. et al. (2013). «Passenger vehicles. Industry and Trade Summary». United States International Trade Commission. Office of Industries. Publication ITS-09. May 2013. 
[9] COMISIÓN EUROPEA (2009). Automotive industry. Comprehensive analysis of the evolution of the automotive sector in Europe. DirectorateGeneral for Employment, Social Affairs and Equal Opportunities.

[10] COMISIÓN EUROPEA (2012a). CARS 21. High Level Group on the Competitiveness and Sustainable Growth of the Automotive Industry in the European Union. Final Report 2012.

[11] COMISIÓN EUROPEA (2012b). CARS 2020: Action Plan for a competitive and sustainable automotive industry in Europe. COM (2012) 636 final.

[12] COMISIÓN EUROPEA (2014c). European Union, Trade in Goods with China. Directorate General for Trade. 16-04-2014.

[13] DAIMLER (2014). Annual Report, 2013.

[14] DEPARTAMENTO DE COMERCIO DE LOS ESTADOS UNIDOS (2012). US-China Deficit and Automotive Trade. 14-08-2012.

[15] DICKEN, P. (2007). Global shift: mapping the changing contours of the world economy. Sage, London. $5^{\text {th }}$ Edition.

[16] EGGER, H. y Egger, P. (2005). «The Determinants of EU Processing Trade». The World Economy, vol. 28, $n^{\circ}$. 2, february, pp. 147-168.

[16] FEENSTRA, R. y HANSON, G.H. (2001). "Global Production Sharing and Rising Inequality: A Survey of Trade and Wages", NBER Working Papers 8372, National Bureau of Economic Research.

[17] FERNÁNDEZ, J.A. y SHENGJUN, L. (2007). China CEO: A Case Guide for Business Leaders in China. Wiley Eds.

[18] FIAT SPA (2014). Annual Report, 2013.

[19] FREYSSENET, M. (ed.) (2009). The Second Automobile Revolution. Trajectories of World Carmakers in the XXI Century. Palgrave MacMillan in association with GERPISA, Groupe d'Étude et de Recherche Permanent sur I'Industrie et les Salariés de l'Automobile.

[20] GÖRG, H. (2000): «Fragmentation and Trade: US Inward Processing Trade in the EU». Weltwirtschaftliches Archiv, vol. 136, Issue 3, pp. 403-422.

[21] HAUGH, D.; MOUROUGANE, A. y CHATAL, O. (2010). «The automobile industry in and beyond the crisis». OECD. Economics Department Working Papers $\mathrm{n}^{\circ} .745$, january 2010.

[22] HUMMELS, D.; ISHII, J. y YI, K. (2001): «The nature and growth of vertical specialization in world trade». Journal of International Economics, vol. 54, $\mathrm{n}^{\circ} 1$, junio, pp. 75-96.
[23] JONES, R. W. y KIERZKOWSKI, H. (1990). «The role of services in production and international trade: A theoretical framework. In R. Jones \& A. Krueger (Eds.)", The political economy of international trade: Essays in honour of Robert $E$. Baldwin.

[24] KIMURA, F. y ANDO, M. (2005). «Two-dimensional fragmentation in East Asia: Conceptual framework and empirics». International Review of Economics and Finance, vol. 14, pp. 317-348.

[25] KLIER, T.L. y RUBINSTEIN, J.M. (2006). "Competition and Trade in the U.S. Auto Parts Sector», Chicago Fed Letter, $n^{\circ} 222$, January.

[26] KPMG (2014). The UK automotive industry and the EU. KPMG. The Society of Motor manufacturers and Traders.

[27] LENG, X. (2004). China-EU Trade Relations. The period after 1975. Linköping University. Master Thesis.

[28] NAM, K.M. (2011). "Learning through the International Joint Venture: Lessons from the Experience of China's Automotive Sector». Industrial and Corporate Change $20, \mathrm{n}^{\circ} 3$, junio 1, 2011, pp. 855-907.

[29] NICHOLLS, A. (2013): «Exports alone can't save Europe's auto industry». Automotive World, $n^{\circ} 21$, january 2013.

[30] OCDE (2013). «Medium-Run Capacity Adjustment in the Automobile Industry". OECD Economics Department Policy Notes, $\mathrm{n}^{\circ} 21$, november.

[31] OMC (2014). International Trade Statistics 2013.

[32] POSTH, M. (2006). 1,000 Days in Shanghai: The Story of Volkswagen - The First ChineseGerman Car Factory. Wiley.

[33] PSA (2014). PSA Peugeot Citroën 2013 Annual Results.

[34] RENAULT (2014). Annual Report 2013.

[35] SSMT (2014). Motor industry facts 2014. The Society of Motor manufacturers and Traders.

[36] SOHM, S.; MICHAEL-LINKE, B. y KLOSSEK, A. (2009). Chinese companies in Germany. Chances and challenges. Deloitte: Bertelsmann Stiftung.

[37] STURGEON, T. y FLORIDA, R. (2004). «Globalization, deverticalization, and employment in the motor vehicle industry". In M. Kenney y R. Florida (eds.) Locating Global Advantage; Industry Dynamics in a Globalizing Economy. Palo Alto, California: Stanford University Press.

[38] STURGEON, T.; BIESEBROECK J.V. y GEREFFI, G. (2008). "Value chains, networks and clusters: reframing the global automotive industry». Journal of Economic Geography, $n^{\circ} 8$, pp. 297-321. 
[38] STURGEON, T. et al. (2009). «Globalisation of the automotive industry: main features and trends». Int. J. Technological Learning, Innovation and Development, vol. 2, $\mathrm{n}^{\circ} 1 / 2$.

[39] TEIJELO, C. (2009). «La UE y China: Una relación en busca de equilibrio». Boletín Económico de Informacion Comercial Española, $\mathrm{n}^{\circ} 2972$, pp. 21-35.
[40] TURKCAN, K. y ATES, A. (2010). «Structure and Determinants of Intra-Industry Trade in the U.S. Auto-Industry». Journal of International and Global Economic Studies, vol. 2, $\mathrm{n}^{\circ}$ 2, December, pp. 15-46.

[41] VDA (2014). Annual Report. 2013.

[42] VOLKSWAGEN (2014). Annual Report. 2013.

[43] VOLVO CARS (2014). Annual Report. 2013. 
ANEXO

TABLA A.1

SECTOR DEL AUTOMÓVIL. CLASIFICACIÓN SEGÚN CÓDIGOS CUCI

\section{Código CUCI}

781 Automóviles y otros vehíc. automot. diseñados para el transporte de personas

782 Vehíc. automot. para transporte de mercancías y vehíc. automot. para usos especiales

783 Vehíc. automot. de carretera, n.e.p.

784 Partes, piezas y accesorios de los automot. de los grupos 722, 781, 782 y 783

7132 Motores de combustión interna, de émbolo, para la propulsión de los vehículos del capítulo 78, del grupo 722 y de los rubros 744.14 , 744.15 y 891.11

7783 Equipo eléctrico, n.e.p., para motores de combustión interna y vehíc. y partes y piezas

Fuente: OMC. Estadísticas Internacionales de Comercio, 2013.

\begin{tabular}{|c|c|c|c|c|c|}
\hline \multicolumn{6}{|c|}{$\begin{array}{c}\text { TABLA A.2 } \\
\text { VENTAS DE TURISMOS EN CHINA SEGÚN MARCA Y FABRICANTE (2013) }\end{array}$} \\
\hline Ranking & Marca & $\begin{array}{l}\text { Ventas* }^{*} \\
2013\end{array}$ & Ranking & Fabricante & $\begin{array}{l}\text { Entregas }^{\star *} \\
2013\end{array}$ \\
\hline 1 & Volkswagen & 2.395 .696 & 1 & Shanghai GM & 1.542 .559 \\
\hline 2 & Hyundai & 1.030 .808 & 2 & Shanghai Volkswagen & 1.525 .008 \\
\hline 3 & Toyota & 857.749 & 3 & FAW Volkswagen & 1.513 .618 \\
\hline 4 & Nissan & 843.063 & 4 & Beijing Hyundai & 1.030 .808 \\
\hline 5 & Buick & 807.700 & 5 & Dongfeng Nissan & 926.229 \\
\hline 6 & Honda & 729.568 & 6 & Chang'an Ford & 682.686 \\
\hline 7 & Chevrolet & 714.743 & 7 & Great Wall & 627.436 \\
\hline 8 & Ford & 678.951 & 8 & FAW Toyota & 554.661 \\
\hline 9 & Kia & 546.766 & 9 & Dongfeng PSA (DPCA) & 552.073 \\
\hline 10 & BYD & 506.189 & 10 & Geely & 549.393 \\
\hline 11 & Changan & 500.500 & 11 & SAIC-GM-Wuling (SGMW) & 548.984 \\
\hline 12 & Wuling & 448.484 & 12 & Dongfeng Yueda Kia & 546.766 \\
\hline 13 & Chery & 437.044 & 13 & BYD & 506.189 \\
\hline 14 & Audi & 411.730 & 14 & Chang'an & 500.500 \\
\hline 15 & Great Wall & 347.672 & 15 & Chery & 443.944 \\
\hline 16 & Citroen & 280.001 & 16 & GAC Honda & 434.828 \\
\hline 17 & Great Wall Haval & 279.764 & 17 & Dongfeng Honda & 321.216 \\
\hline 18 & Peugeot & 272.072 & 18 & GAC Toyota & 303.088 \\
\hline 19 & Skoda & 231.200 & 19 & SAIC & 230.020 \\
\hline 20 & Suzuki & 229.535 & 20 & BMW Brilliance & 207.327 \\
\hline \multicolumn{6}{|c|}{$\begin{array}{l}\text { * Las ventas no incluyen las importaciones (vehículos fabricados en China). } \\
\text { ** Las cifras incluyen solo turismos de } 2 \text { y } 3 \text { puertas, monovolúmenes y deportivos utilitarios. No se incluyen pickups, ni furgonetas y otros vehículos } \\
\text { comerciales. } \\
\text { Fuente: China Association of Automobile Manufacturers. }\end{array}$} \\
\hline
\end{tabular}


ANÁLISIS DEL MODELO DE INTERNACIONALIZACIÓN EN CHINA DE LOS FABRICANTE EUROPEOS...

\section{ANEXO (continuación)}

\begin{tabular}{|c|c|c|c|c|}
\hline \multicolumn{5}{|c|}{$\begin{array}{c}\text { TABLA A.3 } \\
\text { RANKING MUNDIAL DE EMPRESAS PRODUCTORAS DE VEHÍCULOS (2012) }\end{array}$} \\
\hline Ranking & Empresa & País & Unidades & Porcentaje \\
\hline 1 & Toyota & Japón & 10.104 .424 & 12,0 \\
\hline 2 & General Motors & Estados Unidos & 9.285 .425 & 11,0 \\
\hline 3 & Volkswagen & Alemania & 9.254 .742 & 11,0 \\
\hline 4 & Hyundai & Corea del Sur & 7.126 .413 & 8,5 \\
\hline 5 & Ford & Estados Unidos & 5.595 .483 & 6,7 \\
\hline 6 & Nissan & Japón & 4.889 .379 & 5,8 \\
\hline 7 & Honda & Japón & 4.110 .857 & 4,9 \\
\hline 8 & PSA & Francia-China & 2.911 .764 & 3,5 \\
\hline 9 & Suzuki & Japón & 2.893 .602 & 3,4 \\
\hline 10 & Renault & Francia-China & 2.676 .226 & 3,2 \\
\hline 11 & Chrysler & Estados Unidos & 2.371 .427 & 2,8 \\
\hline 12 & Daimler AG & Alemania & 2.195 .152 & 2,6 \\
\hline 13 & Fiat & Italia & 2.127.295 & 2,5 \\
\hline 14 & BMW & Alemania & 2.065 .477 & 2,5 \\
\hline 15 & SAIC & China & 1.783 .548 & 2,1 \\
\hline 16 & Tata & India & 1.241 .239 & 1,5 \\
\hline 17 & Mazda & Japón & 1.189 .283 & 1,4 \\
\hline 18 & Dongfeng motor & China & 1.137 .950 & 1,4 \\
\hline 19 & Mitsubishi & Japón & 1.109 .731 & 1,3 \\
\hline 20 & Changan & China & 1.063 .721 & 1,3 \\
\hline
\end{tabular}


